\title{
METAMATERIAL ENHANCED FLUORESCENCE DETECTION
}

Sean J. Molesky

Electrical and Computer Engineering, University of Alberta

In this article I show how materials created from designer functional units much smaller than the wavelength of operation , or metamaterials, can be used to decrease the lifetime of fluorescence based emitters. This is goal accomplished in three parts. First, the fundamental physical equations describing both fluorescent emission and the particular class of metamaterial required will be over viewed in a broad two part introduction. Next, making use of a seldom seen approach, I will present the Green's functions formalism of spontaneous emission of a quantum emitter above a material slab. Finally, I will present devices to reduce the lifetime of quantum emitters that could provide a large resolution enhancement for fluorescence based sensors, focussing on the wavelengths of 600 and $800 \mathrm{~nm}$.

\section{INTRODUCTION AND MOTIVATION}

Considering the ubiquity of quantum emitters in both device application and understanding throughout all branches of the natural sciences, it should not be surprising to learn just how much research has gone into the prospect manipulating their behavior in the hopes of producing superior device performance [1, 2]. Yet, despite the best efforts of the intellectual giants of the field, until incredibly recently a satisfactory method for significant broadband manipulation of emitter lifetime, the key parameter governing the properties of quantum emitters, had not been found [3].

To gain a better understanding of what this parameters means, we begin by recalling Fermi's golden rule:

$$
R_{s}=\frac{2 \pi}{\hbar}\left|\left\langle\Psi_{f}\left|H_{\text {int }}\right| \Psi i\right\rangle\right|^{2} n\left(\epsilon_{f}\right)
$$

Where $\Psi$ represents the intial quantum state, $n\left(\epsilon_{f}\right)$ the final density of states and $\mathrm{H}$ the Hamiltonian of the system [4].

This equation allows us to see that the lifetime of an excited state relies heavily on the final density of states which the quanta of energy may occupy. For fluorophores, the examples of interest in this article, this means the lifetime of the excited vibrational state is determined by the states that the released photon could possibly occupy upon emission. Or, from a perhaps more instinctively strange anthropomorphic perspective, a photon is much more likely to come into being if its feelers find many places where could fit in. With this somewhat odd reflection in mind, engineering the lifetime of fluorophores, or any other quantum emitter, is a matter of altering the number of states a photon of a given frequency could exist in, or more concisely the photonic density of states.

Conventionally, the primary means for producing the desired enhancement to the photonic density of states has

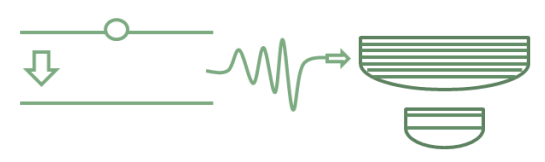

Fig. 1 Lifetime of the Excited State is Based on the Density of States Available to the Emission

Fig. 2 Cavity to Reduce Emitter Lifetime Via the Purcell Effect Michler et. al been through the use of resonator cavities, based on the ingenious work of noble prize winning physicist Edward Purcell. While perhaps overly simplistic, the effect bearing Purcell's name can be understood almost entirely from considering an argument about emitted power ${ }^{1}$ :

Given that it is possible to couple power from a photon emitter into the resonant modes of a cavity above and beyond what is possible in free space, provided there is an overlap between the bandwidth of the emission and that of the resonant mode [5]. In equal measure, it makes intuitive sense that an increase in the net power output must be directly tied to a reduction of the lifetime of any excited state from which photon emission occurs. Therefore, resonant cavity modes must cause an enhancement to the photonic density of states, and in turn offer an engineering solution to achieving control over devices based on quantum emitter .

Unfortunately, in practical application the resonant cavity approach is severally limited by the typically minuet bandwidth of resonant cavity modes in comparison to that of room temperature photonic emitters [6]. In order to produce a meaningful enhancement of the photonic density of states, required to get the best possible results from sensing and detection based devices, a new approach must be taken. This is no easy task in view of the possibilities presented by standard materials.

However, recent results suggest that this does not mean a new solution to broadband augmentation of the photonic density of states is not possible. The maturation of nanostructure fabrication techniques has turned many fantasies into practical standard recipes, and its a good guess to assume that once again it will play a leading role [7]. Using arrays of designer nanostructures, metamaterials with properties above and beyond anything found in nature can now be produced and functionally implemented with a high degree of precision; creating a new paradigm for a host of long standing engineering problems. As it currently appears, the problem of how to alter the photonic density of states can certainly be included in this category.

\section{UNDERSTANDING HYPERBOLIC METAMATERIALS Isofrequency Surfaces and Maxwell's Equations \\ Perhaps the most efficient way to understanding the hyperbolic metamaterial solution to enlarging the photonic density of states is to return to the fundamental electromagnetic properties necessitated by Maxwell's}

\footnotetext{
1. A thorough derivation of the Purcell effect is fairly involved and will not be dealt with in this article.
} 
equations [8]:

$$
\begin{gathered}
\vec{\nabla} \cdot \vec{D}=\rho_{f} \\
\vec{\nabla} \times \vec{E}=-\frac{\partial \vec{B}}{\partial t} \\
\vec{\nabla} \cdot \vec{B}=0 \\
\vec{\nabla} \times \vec{H}=\vec{J}_{f}+\frac{\partial \vec{D}}{\partial t}
\end{gathered}
$$

Assumming plane wave solutions of the form:

$$
E(\vec{r}, t)=A_{0} R e\left(e^{i(\vec{k} \cdot \vec{r}-\omega t)}\right)
$$

Where $\mathrm{k}$ the wave vector with a magnitude defined by $\frac{2 \pi}{\lambda}$. Taking a simple approach, this quantity can be understood as a measure which is inversely proportional to wavelength that dictates the propagation direction of the plane wave by (6). If the curl of (5) is taken, in conjunction with the result of (3), in a linearly polarisable material free of charges and currents it can be found that:

$$
\frac{k_{x}^{2}}{\epsilon}+\frac{k_{y}^{2}}{\epsilon}+\frac{k_{z}^{2}}{\epsilon}=\frac{\omega^{2}}{c}
$$

Which, if the frequency is fixed, has a form equivalent to the mathematical description of a sphere.

We can now easily develop a graphical understanding of the photonic density of states. Given that each possible photonic state can be represented in wave vector space as a small cube of some dimension, dictated by the properties of the material in which the surface is plotted, the photonic density of states can be pictured as the amount of volume that would be added if the sphere were given an infinitesimal thickness, and turned into a shell, through an infinitesimal addition to the band of its frequency (7). This strictly geometric interpretation depicts a number of very important realizations. In particular, it shows the number of photonic states must be fixed as the volume of an added shell is always finite ${ }^{2}$.

\section{Hyperbolic Metamaterials}

Bearing this in mind, we can now begin to consider more complicated scenarios. If the dielectric constant of (7) were directional and of undefined sign, then (7), following the treatment descried in the beginning of this section, would be transformed into

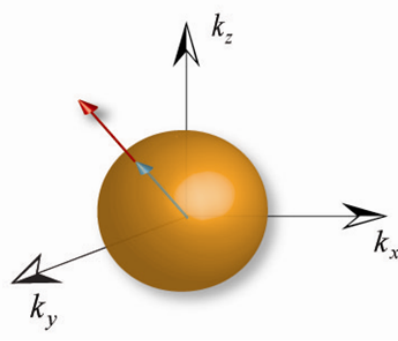

Fig 3. Isofrequency Surface of the Free Space Dispersion Relation

$$
\frac{k_{x}^{2}}{\epsilon_{x}} \pm \frac{k_{y}^{2}}{\epsilon_{y}} \pm \frac{k_{z}^{2}}{\epsilon_{z}}=\frac{\omega^{2}}{c}
$$

Which serves as the generating equation for a vast range of conic surfaces. In particular, if in two directions the dielectric were of positive sign and in the other negative, technically known as an extreme anisotropy in the dielectric tensor ${ }^{3}$, the relation of a hyperboloid would result. Returning briefly to the graphical scheme presented earlier in this section, if such a dispersion relation were possible then an unbounded density of states would be produced by virtue of the unbounded wave vector magnitude describe by:

$$
\frac{k_{x}^{2}}{\epsilon_{\perp}}+\frac{k_{y}^{2}}{\epsilon_{\perp}}-\frac{k_{z}^{2}}{\epsilon_{\|}}=\frac{\omega^{2}}{c}
$$

As has recently been explored in the context of creating a hyperlens [9], a certain class of metamaterials, known as hyperbolic metamaterials, are capable of displaying

this exact behaviour. With a reasonable concept of the basis of the metamaterial solution firmly in place, our attention may now turn to how such materials may be fabricated, and a more rigorous description of their exact effects on photonic emitters.

\section{Effective Medium Theory}

On the macroscopic scale, the means for producing hyperbolic metamaterials, and thus gaining improved control over the decay rate of fluorophores, is strongly tethered to the averaging procedures ${ }^{4}$ which describes the reaction of an electromagnetic wave to sub wavelength features. If we consider a stack of materials with dielectric constants of alternating magnitude, it is not difficult to show that the relations imposed by Maxwell's equations (2) between the electric flux density, $\vec{D}$, and fields, $\vec{E}$, of the alternating slabs are given by:

$$
\begin{aligned}
\vec{D}_{1 / 2} & =\epsilon_{1} \vec{E}_{1 / 2} \\
E_{1}^{\|} & =E_{2}^{\|}
\end{aligned}
$$

2. The diffraction limit arises as the maximum magnitude of the wave vector, for propagating waves, is bound by the isofrequency sphere. Were one of the wave vector components larger than the fixed radius dictated by (7) one of the other two wave vector components would have to be imaginary, resulting in a decaying or evanescent wave through (6). Isofrequency surfaces may also be used to show why emission in hyperbolic metamaterial must be directional. As energy must always flow in the direction of group propagation, which through the gradient is always perpendicular to the iso-surface, for any particular hyperbolic metamaterial there will be a perferred direction for energy flow.

3. A tensor is an array of the second rank which maps the directionality of the dielectric into a form that can be used in conjunction with Maxwell's equations (2-5).

4. For objects much smaller than the wavelength of light at a given frequency the bulk or macroscopic behavior can be well modeled by assuming the small objects form an effective medium. One of the more popular procedures for determining the parameters of this medium known as the Maxwell-Garnett theory. 


$$
D_{1}^{\perp}=D_{2}^{\perp}
$$

Which, if the wavelength of the electromagnetic perturbation is sufficiently large in comparison to the thickness of a few layers, such that it may be assumed that $\vec{D}$ and remain constant over the interval, give rise to the effective media descriptions:

$$
\begin{gathered}
\epsilon^{\|}=\epsilon_{1}(P)+\epsilon_{2}(1-P) \\
\frac{1}{\epsilon^{\perp}}=\frac{P}{\epsilon_{1}}+\frac{(1-P)}{\epsilon_{2}}
\end{gathered}
$$

Where $\mathrm{P}$ represents a fill factor based on the proportion of one of the two alternating slabs to the other, $\frac{d 1}{d 1+d 2}$.

Looking carefully at these equations and recalling the result of (8), it can be seen that if one of the dielectrics is given a positive magnitude, which in the context of dielectrics will be referred to as a dielectric, and the other a negative magnitude, characteristic of metals, that the desired hyperbolic dispersion relation would result. Despite the seeming superficiality of this argument, the result is quite accurate provided that all of the assumptions made in its construction are satisfied. To implement a hyperbolic metamaterial all that is needed is defined layers of alternating metal and dielectric of the correct thickness.

\section{Surface Plasmon Polariton (SPP)}

While the effective medium approach is an extremely useful description of the macroscopic characteristics of a hyperbolic metamaterial (HMM), it provides little explanation of how these phenomena arrive from the nanosized building blocks. Thus, in order to have a firm enough grasp of the concepts involved in creating HMM to move on to a satisfactory exploration of their effects on fluorophores it is crucial to also understand the surface plasmon polariton state.

Directly, a surface plasmon polariton (SPP) mode is as quanta of energy trapped in an oscillatory mode which couples the free electron density at a surface to incoming photons. Or, in turning to the mathematical sophistication of Maxwell's equations, as a TM electromagnetic boundary wave solution described by:

$$
k_{x}=\frac{\omega}{c}\left(\frac{\epsilon_{1} \epsilon_{2}}{\epsilon_{1}+\epsilon_{2}}\right)^{1 / 2}
$$

(Found by guessing at the existence of a state characterized by a magnetic field perpendicular to the plane and an electric field in the other two perpendicular directions ${ }^{5}$ and an application of the boundary conditions from Maxwell's equations. $)^{6}(10)(11)$

While the general shape and field confinement of the SPP solution is interesting in and of itself, it is in applying the Drude model:

$$
\epsilon_{2}(\omega)=1-\omega_{p}^{2} / \omega^{2}
$$

where $\omega_{p}$ is a material property known as the plasma frequency, (8) to (15) that truly exciting behaviour occurs.

From the preceding plot of the SPP dispersion relation it can be seen that at the frequency $\frac{\omega_{p}}{\sqrt{2}}$ the over all behaviour of wave vector frequency relation becomes

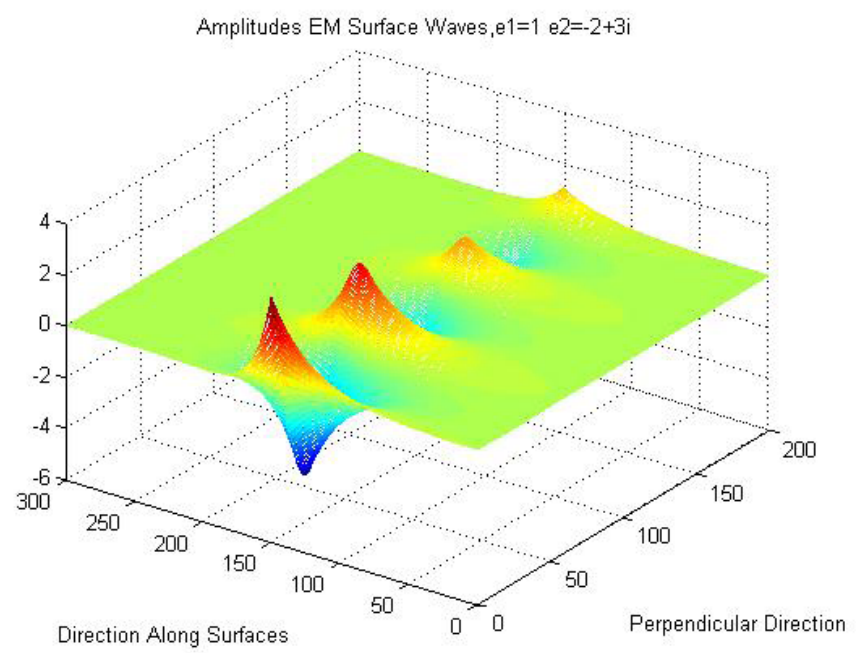

Fig. 5. Plasmon Polaritron for Lossy Metal

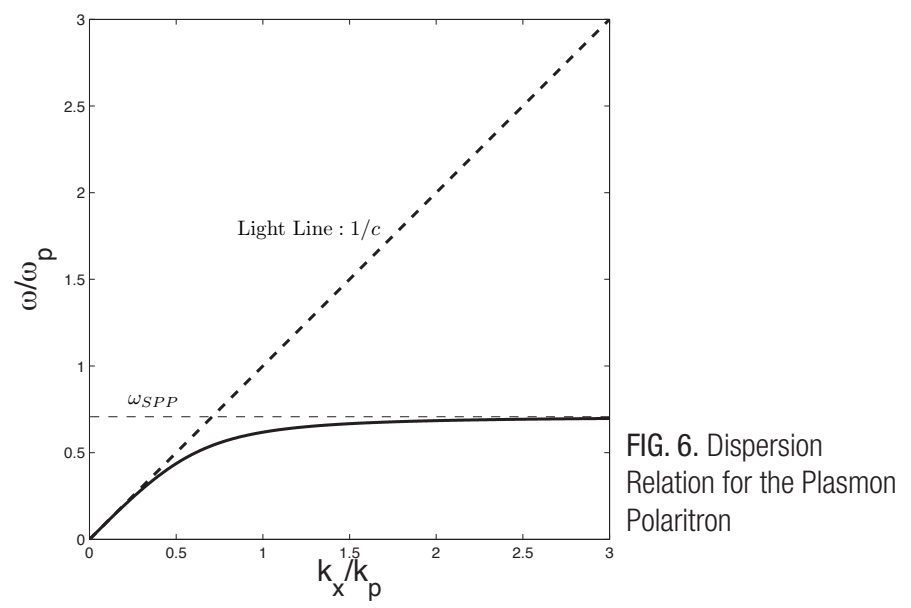

asymptotic; meaning that a photon coupled to the free electron density at the surface of a metal could once again access an essentially unlimited number of states by virtue of the unbounded magnitude of its wave vector. This is no coincidence, it turns out that through coupling to the SPP modes which exist simultaneously at each metal dielectric interface that the bulk properties of a HMM can be recovered. As these views are inherently interreliant, this consideration also gives an inkling to which metals are best suited for hyperbolic metamaterial implementations ${ }^{7}$. We are now ready to pursue the mathematical treatment required to more fully comprehend fluorophore lifetime in the presence of hyperbolic metamaterials.

\section{MATHEMATICAL TREATMENT FOR CALCULATING LIFETIME NEAR METAMATERIALS}

Green's Function Method

We will now derive a rather novel Green's function

5. Electromagnetic fields with this type of orientation are more commonly referred to as transverse magnetic, or TM solutions.

6. Historically, the idea of a SPP was introduced by Zenneck and Sommerfeld to acount to the extra energy observed in radio transimissions compared to the expected free space values.

7. Based off this discussion it would seem that any metal with a strong SPP mode would be a good candidate.

8. The Green's function method for solving differential equations is based on the response of a system to a point source. This solution can then be moved and weighted to solve the problem for more complicated sources. 
approach to determine the lifetime of a quantum emitter, building on two primary considerations. First, as the Green's function method 8 of solving partial differential equations is at its deepest level a complete description of the correlation between the sources and responses of a given system; a more thorough understanding of the underlying physics of a problem can generally be gleamed through the Green's Function solution. Secondly, a proper mathematical description of the emitted power requires that we consider free space decaying waves which in general cannot be handled by first order perturbation theory.

\section{General Form}

Through an examination of the Green's function solution to the Helmholtz equation, we will finally assemble the necessary mathematical machinery to reach our final theoretical task of determining fluorophore lifetime above a metamaterial.

$$
\begin{gathered}
\left(\nabla^{2}+k^{2}\right) \psi=\frac{2 m}{\hbar^{2}} V \psi \\
k=\frac{2 m E}{\hbar^{2}} \\
\left(\nabla^{2}+k^{2}\right) g\left(r, r^{\prime}\right)=\delta(r)
\end{gathered}
$$

Now, setting $r^{\prime}=0$ and transforming the problem into wave vector space (q), we get:

$$
\begin{gathered}
g(r)=\iiint \frac{d^{3} q}{(2 \pi)^{3}} g(q) e^{i \vec{q} \cdot \vec{r}} \\
\delta(r)=\iiint \frac{d^{3} q}{(2 \pi)^{3}} e^{i \vec{q} \cdot \vec{r}} \\
\iiint\left(\nabla^{2}+k^{2}\right) \frac{d^{3} q}{(2 \pi)^{3}} g(q) e^{i \vec{q} \cdot \vec{r}}=\iiint \frac{d^{3} q}{(2 \pi)^{3}} g(q) e^{i \vec{q} \cdot \vec{r}}
\end{gathered}
$$

Which must be true for all $r$, so:

$$
g(q)=\frac{1}{k^{2}-q^{2}}
$$

This equation, if we displace the poles from the real axis and make a transformation to polar coordinates, can be solved by making use of Cauchy integral theorem, leading to the famous Lippmann-Schwinger equation:

$$
\begin{gathered}
g(r)=-\frac{e^{i k r}}{4 \pi r} \\
\Psi=\Psi_{o}-\int \frac{e^{i k\left|r-r^{\prime}\right|}}{4 \pi\left|r-r^{\prime}\right|} \frac{2 m}{\hbar^{2}} V\left(r^{\prime}\right) \psi d V^{\prime}
\end{gathered}
$$

Which additionally, by making the ansatz that $\psi_{o}=e^{i \vec{k} \cdot \vec{r}}$, is also the general equation of a scattering potential.

$$
\Psi=\Psi_{o}-\int \frac{e^{i k\left|r-r^{\prime}\right|}}{4 \pi\left|r-r^{\prime}\right|} \frac{2 m}{\hbar^{2}} V\left(r^{\prime}\right) e^{i \vec{k} \cdot \vec{r}} d V^{\prime}
$$

\section{Maxwell's Equations and Green's Functions}

Our next undertaking is to cast Maxwell's equations in the form of the Helmholtz equation in order to make use of the results of the previous subsection. Using the fact that the electromagnetic vector potential [8] may be defined as:

$$
\begin{gathered}
\vec{\nabla} \times \vec{A}=\vec{B}^{\prime} \\
\vec{E}=-\frac{1}{c} \frac{\partial \vec{A}}{\partial t}+\vec{\nabla} \phi
\end{gathered}
$$

Maxwell's equations can be used to create a Helmholtz equation for the vector potential, and in turn the electric field, in linearly polarisable media by taking taking the cross product of (26) and using the results of (5) and (27) in the Lorentz guage leading to:

$$
\nabla^{2} \vec{A}-\frac{\mu \epsilon}{c^{2}} \frac{\partial^{2} \vec{A}}{\partial t^{2}}=\frac{-4 \pi \mu \vec{J}}{c}
$$

Which, in Cartesian coordinates, through the use of the previous subsection and the well known Wyle identity has a Green's function form of:

$$
g(r)=\frac{i}{8 \pi^{2}} \int_{-\infty}^{\infty} \frac{\partial k_{x} \partial k_{y}}{k_{z}} e^{i\left(k_{x} x+k_{y} y+k_{z} z\right)}
$$

related to the Green's Function of the electric field by

$$
G^{\leftrightarrow}=\left[I^{\leftrightarrow}+\frac{\vec{\nabla} \cdot \vec{\nabla}}{k_{1}^{2}}\right] g(r)
$$

Where the symbols are tensors and $k_{1}=\frac{\sqrt{\epsilon \mu}}{c}$.

Power and Final Results

Recalling the fundamental electrodynamic result that:

$$
\langle P\rangle=\int \frac{1}{2} \operatorname{Re}(\vec{J} \cdot \vec{E}) d V
$$

The output power of a quantum emitter in free space, making use of the Green's Function approach presented earlier, can be determinedto be ${ }^{9}$ :

$$
\langle P\rangle=\frac{n \mu \omega^{4}}{3 c^{3}}|\xi|^{2}
$$

Where $\mathrm{n}$ is the material index, $\mu$ the magnetic permeability and $\alpha$ the dipole moment of the photonic emitter.Note that this is a correction to the standard Larmor formula of $\langle P\rangle=\frac{n \omega^{4}}{3 c^{3}}|\xi|^{2}$ previously only observed following arguments from quantum electrodynamics. Taking this result and making use of the semi-classical result based on Fermi's golden rule that:

$$
\Gamma=\frac{P}{\hbar \omega}
$$

Where $\Gamma$ is the photonic density of states. If we were to go back through the procedures presented in this section with the amendment that

$$
G^{\leftrightarrow}=G_{\text {emitted }}^{\leftrightarrow}+G_{\text {reflected }}^{\leftrightarrow}
$$

We would come to the final conclusion that in the presence of a material slab the emitted power, inversely proportional to lifetime, is given by:

$$
\frac{\Gamma}{\Gamma_{o}}=\frac{\tau_{o}}{\tau}=1+\frac{3}{2} \operatorname{Re}\left[\int_{0}^{\infty} \frac{r_{p}(s) S^{3} e^{2 i s_{z} K_{0} * d}}{s_{z}} d s\right]
$$

Where $s^{2}=\frac{k_{x}^{2}+k_{y}^{2}}{K_{0}^{2}} s_{z}^{2}=\frac{k_{z}^{2}}{K_{0}^{2}}, \mathrm{~d}$ is the thickness of the slab, the reflection coefficient of the incident $p$-polarized electromagnetic wave and $\tau$ the lifetime.

9. In this derivation it was assumed that the dipole emitter is pointing up out of the material slab. 


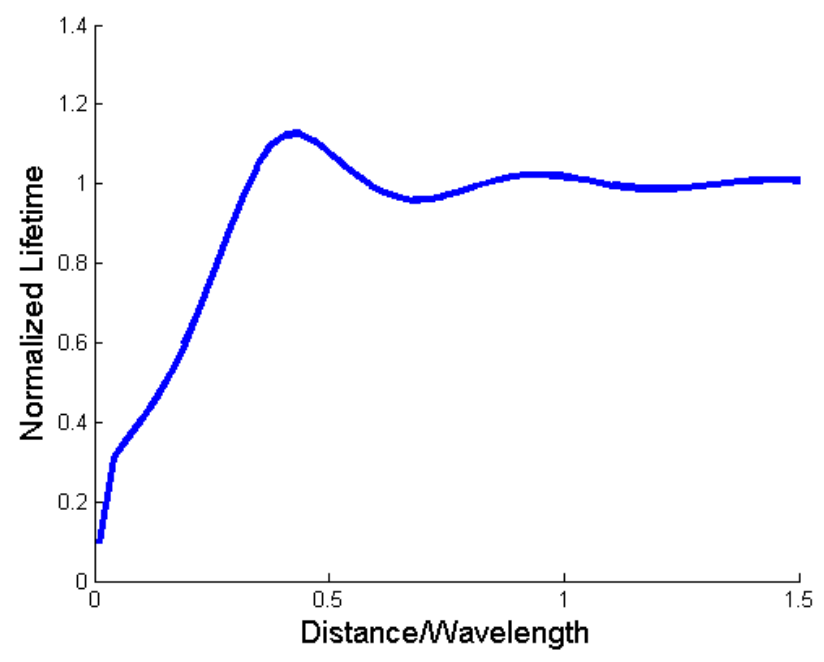

Fig. 7. Sample Quantum Emitter Lifetime Above a Metallic Slab

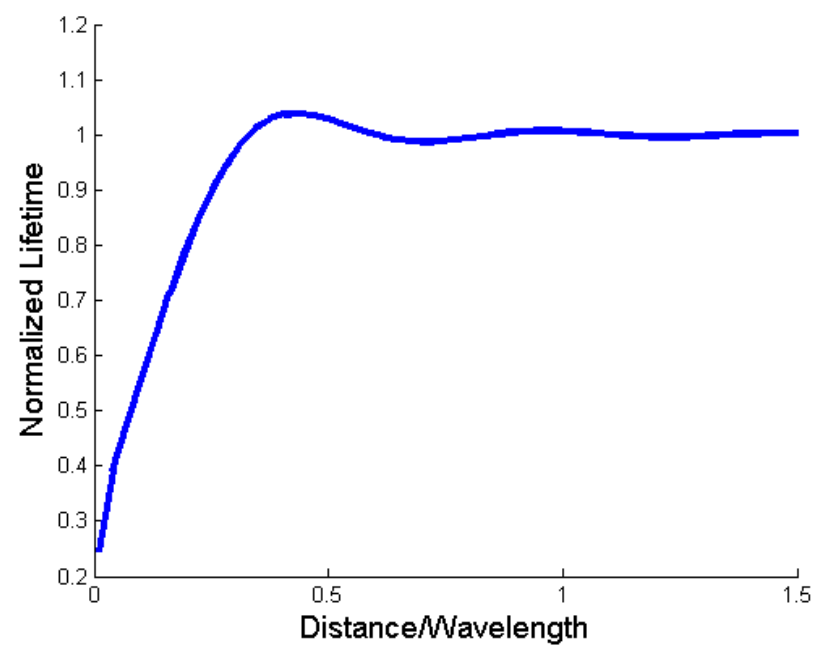

Fig. 9. Sample Quantum Emitter Lifetime Above a Dielectric Slab

\section{CONCLUSIONS}

\section{Lifetime near a Homogeneous Slabs}

Having completed our tour of the theoretical principles involved in calculating the lifetime of a quantum emitter in the presence of any type of material, we can now turn to the final results.

For materials with negative dielectric constants, as the fluorophore descends closer and closer to the interface

more power begins to couple to the plasmonic mode offering some degree of control over the lifetime of the fluorophore. However, as can be seen in the power spectrum plot, the dominant contributions to the enhancement of the photonic density of states are coming from coupling to lossy non radiative modes, a phenomenon known as quenching ${ }^{10}$. As this emitted power may not be extracted from the material for detection the situation is less than ideal.

Materials with strictly positive dielectric constants, as initially predicted, also fail to provide the needed photonic density augmentation to provide a suitable solution to engineering the lifetime of fluorophores. As can be seen from the power spectrum graph of a model dielectric (seen above), the decrease in photonic lifetime in the dielectric case is due to the resonant Fabry-Perot modes

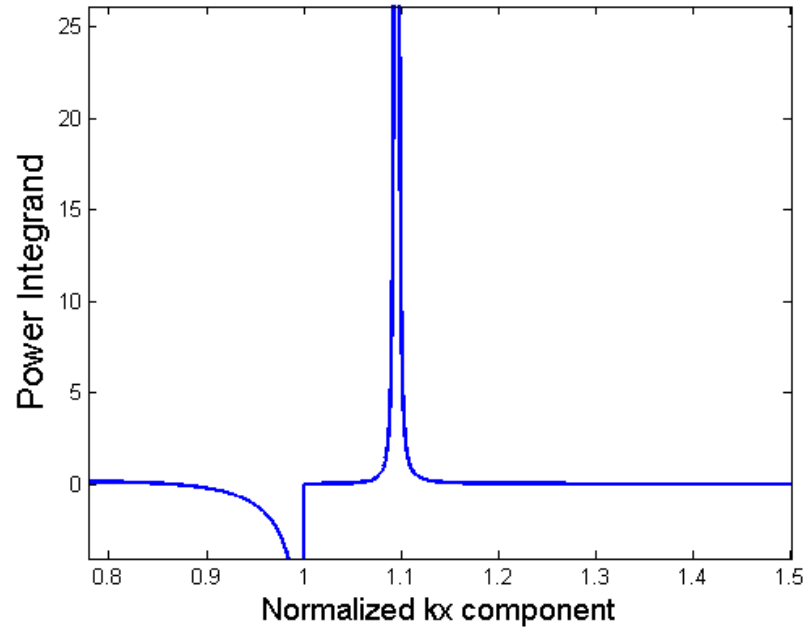

Fig. 8. Sample Quantum Emitter Power Spectrum Above a Metallic Slab

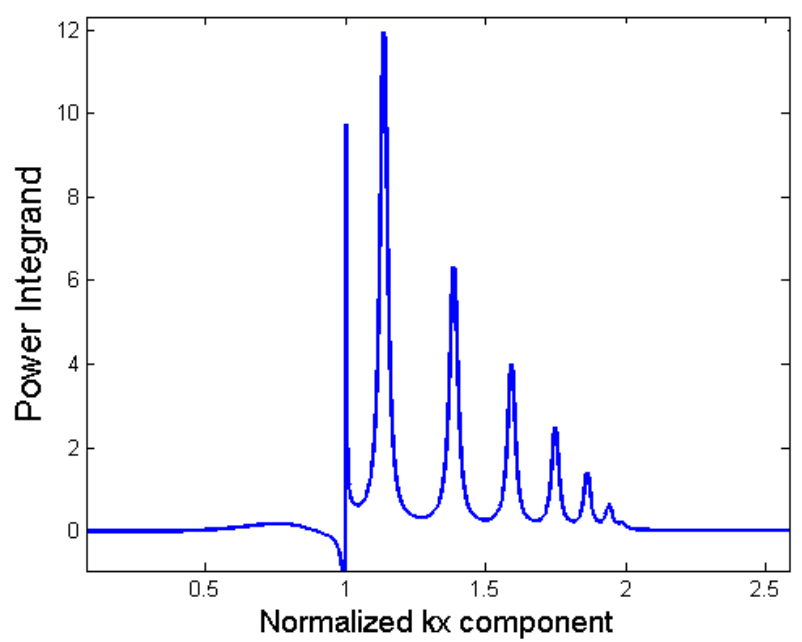

Fig.10. Sample Quantum Emitter Power Spectrum Above a Dielectric Slab

of the slab producing a increase in the photonic density of states via the Purcell effect. As previously discussed in the introduction of this report, this is an unsatisfactory solution in several regards.

\section{Lifetime near Metamaterial Slabs ${ }^{11}$}

Contrary to the plots produced by a homogeneous slab, the modeled behaviour of a quantum emitter above a metamaterial provides significant lifetime reduction with the dominant portion of the power coming from the coupling into surface plasmon modes. With this conformation of our intial inquiry we are ready to produce first order designs of hyperbolic metamaterials for sensing applications.

\section{Designing Metamaterials for Fluorophore Sensing}

Creating the best initial design for HMMs to enhance fluorescence based detection beings with choosing the proper materials. Drawing from the theoretical investigation of the underlying physics of hyperbolic metamaterials, we

10. This can be determined graphically by noting that only one fairly narrow peak exists, yet the lifetime drops quite quickly.

11. The difference between type one and two begin which of the dielectric tensor components is negative. In type 1 this is parallel component; type 2 the perpdicular. 


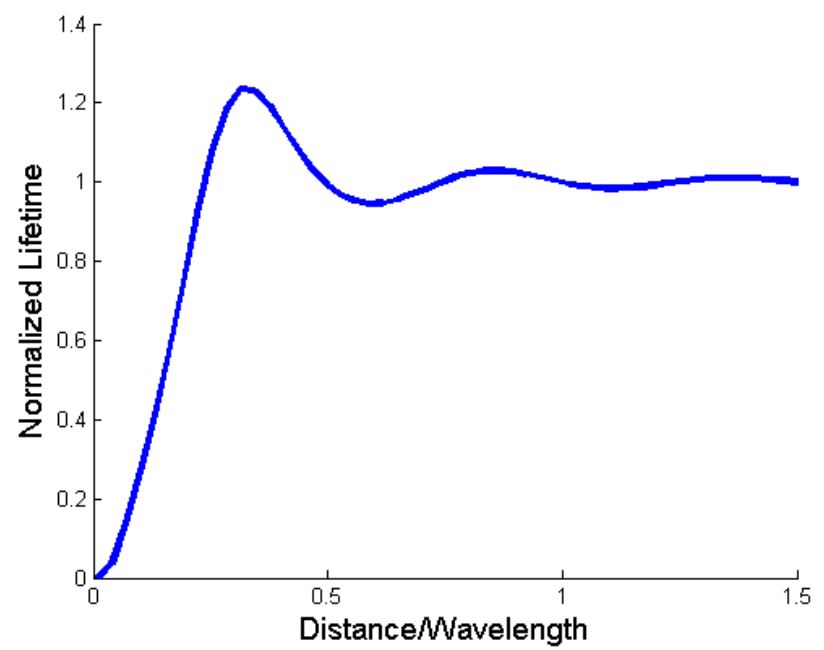

Fig.11. Sample Quantum Emitter Lifetime Above a Slab of Type 1 HMM

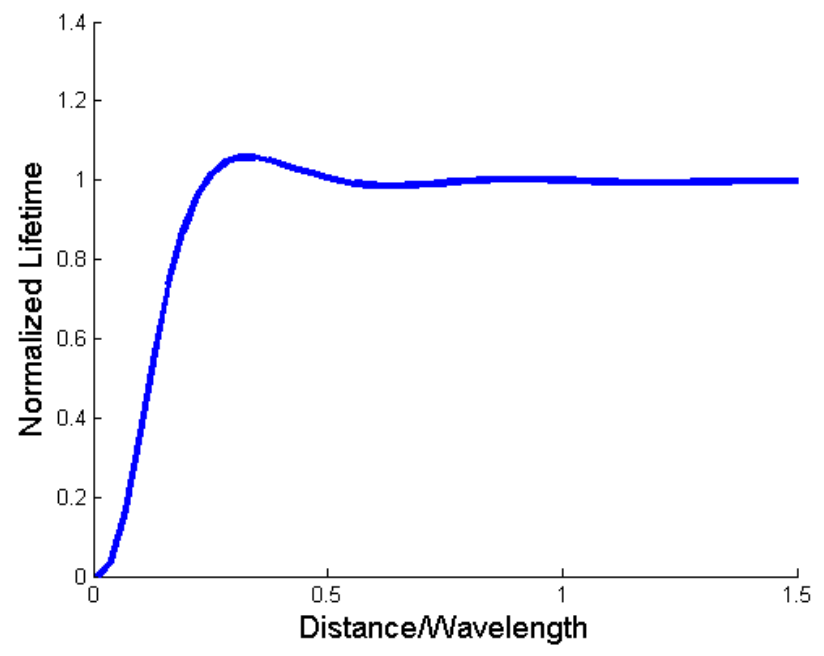

Fig. 13. Sample Quantum Emitter Lifetime Above a Slab of Type 2 HMM

can deduce that in part this amounts to choosing the metal with the smallest imaginary dielectric component, and a complementary dielectric to create reasonable fill factors (14) in the ranges of interest. However, also of concern is the fact that these designs are meant to eventually to provide a basis for creating physical devices, and that as such only materials and procedures which could be accessed by the average high end fabrication facility should be proposed. With these considerations in mind, a careful search dictated by material parameters leads us to focus on Silver, due to it having the smallest imaginary dielectric component as well as favourable properties for fabrication, and $\mathrm{TiO} 2$ for the reasons mentioned above ${ }^{12}$.

The fill factor for these materials can then be calculated at the wavelengths of interest ${ }^{13}$ by setting the value of the dielectric parallel to the interface equal to negative one, in order to provide the best possible coupling as predicted by Maxwell's equations. Leading to the following results:

Taking these results and performing a similar procedure to the work of Joseph Lakowicz [11], leads to us to the conclusion that it is reasonable to assume that HMM designs will offer at least an order of magnitude increase above and beyond the resolution of state of the art silver fluorescence based sensors. Considering the ease

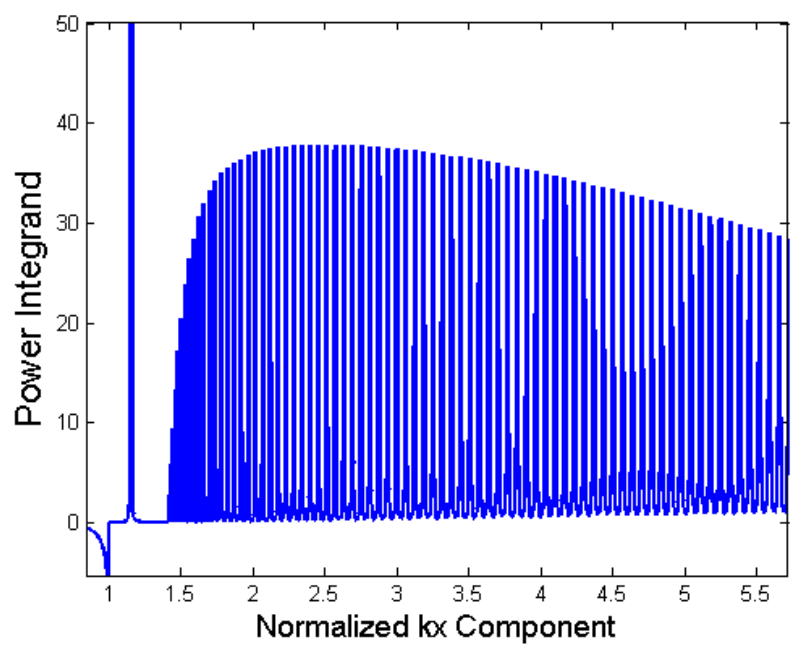

Fig.12. Sample Quantum Emitter Power Spectrum Above a Slab of Type 1 HMM

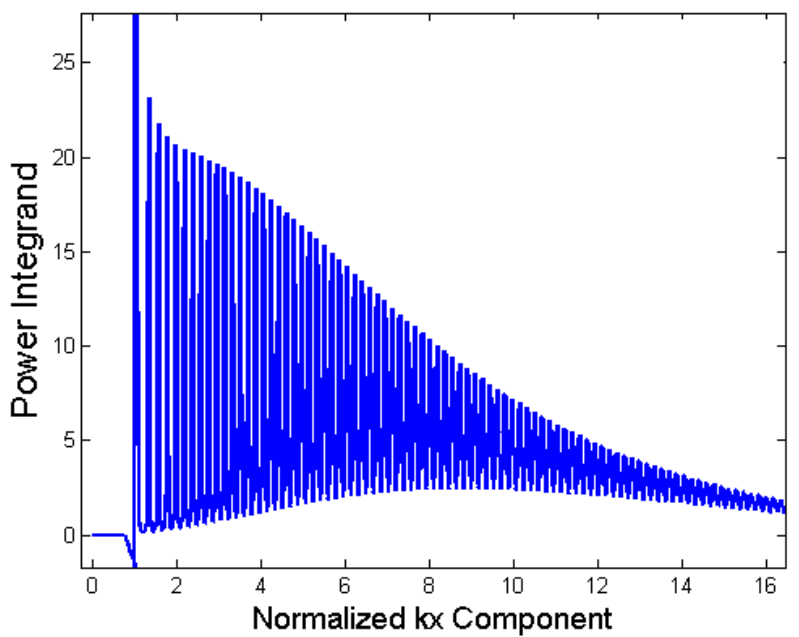

Fig.14. Sample Quantum Emitter Power Spectrum Above a Slab of Type 2 HMM

with which HMM systems can be designed, commerical application is probable and possibly already in progress.

\section{CONCLUSION}

In this article I have presented the basis for understanding how the lifetime of a quantum emitter can be manipulated through the use of hyperbolic metamaterials. Although the details of how a reduced lifetime corresponds to enchanced resolution is left to other authors [12], the basic idea of a flash begin easier to detect than a constant low level source should be reason enough to believe that this is indeed the case. If you have any questions about the article, or would like more information on any of the topics presented, please feel free to email address listed at the beginning of the article.

12. Silver may be deposited down to approximately $11 \mathrm{~nm}$ by sputtering and in thinner films by atomic layer deposition[10]. Ti02 can be deposited over silver ether by chemical vapour deposition or by once again using atomic layer depotion.

13. Six hundred and eight hundred nanometer wavelengths were chosen due to the large number of commonly used fluorophores in these regions, such as QDOT 800 and AlexaFluor 555 (source: Invtrogen). 


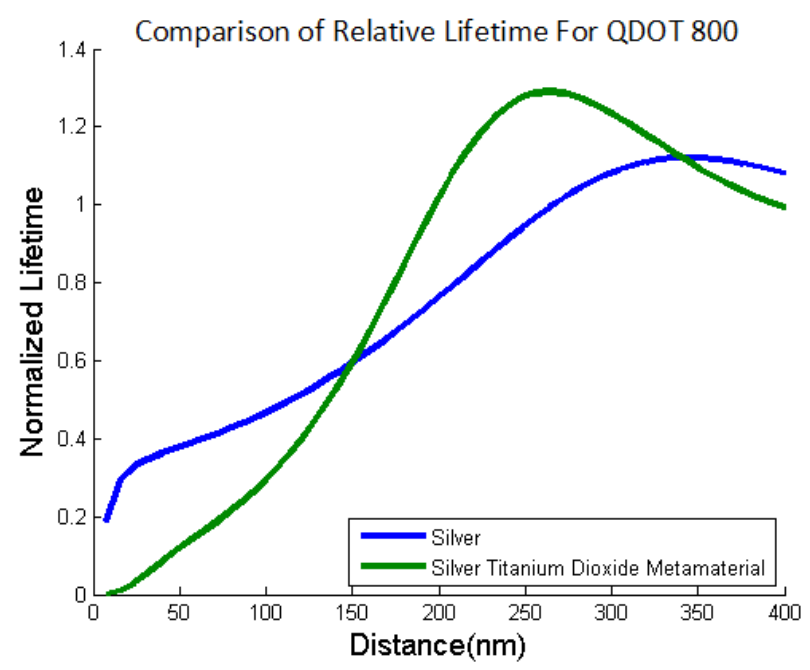

Fig. 15. Comparison of Lifetime Between HMM Design and Current Plasmonic Enhanced Slab at 800nm

\begin{tabular}{|l|l|}
\hline Material & Reflectance Average [\%] \\
\hline Silver $(800 \mathrm{~nm})$ & 86.9 \\
\hline Silver $(600 \mathrm{~nm})$ & 73.6 \\
\hline HMM $(800 \mathrm{~nm})$ & 19.8 \\
\hline HMM $(600 \mathrm{~nm})$ & 19.8 \\
\hline
\end{tabular}

Table 1. Comparison of Reflectance between Metamaterial Design and Current Plasmonic Enhanced Slab

\section{ACKNOWLEDGMENTS}

The work presented in this article was completed as part of a Capstone Design Project in the department of Electrical and Computer Engineering. The author would like to acknowledge Professors Zubin Jacob and Chris Backhouse for their time and guidance throughout the project; and Ward Newman for many insightful discussions.

\section{Reference}

[1] J. M. Gerard and B. Gayral. Strong Purcell effect for InAs quantum boxes in three-dimensional solidstate microcavities. Journal of Lightwave Technology, 17(11):2089-2095, 1999.

[2] S. Noda, M. Fujita, and T. Asano. Spontaneous-emission control by photonic crystals and nanocavities. Nature Photonics, 1(8):449-458, August 2007.

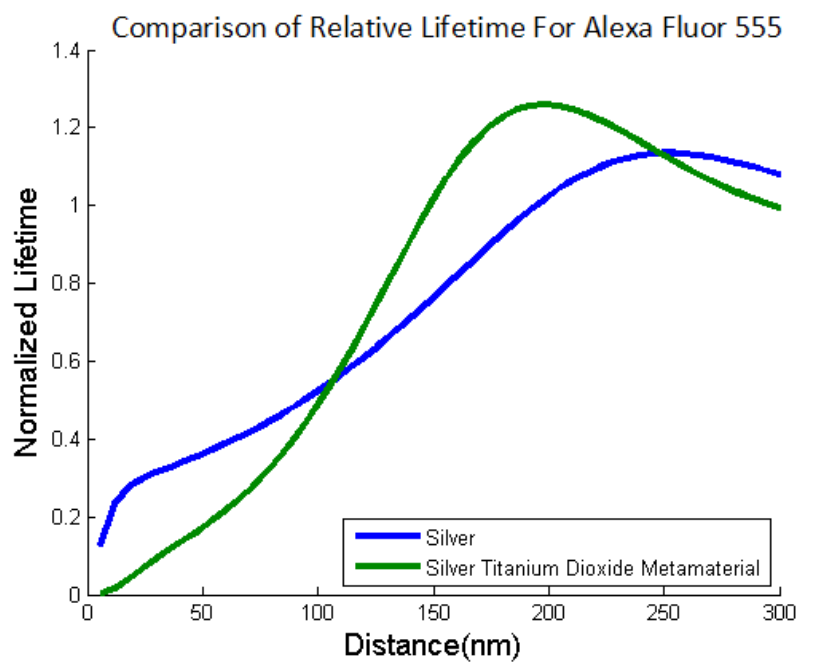

Fig.16. Comparison of Lifetime Between HMM Design and Current Plasmonic Enhanced Slab at 600nm

[3] Z. Jacob, I. Smolyaninov, and E. Narimanov. Broadband Purcell effect: Radiative decay engineering with metamaterials. ArXiv, 0910.3981, October 2009.

[4] L. E. Ballentine. Quantum Mechanics: A Modern Development. World Scientific, Hackensack, NJ, 1998.

[5] J. Gerard, B. Sermage, B. Gayral, B. Legrand, E. Costard, and V. Thierry-Mieg. Enhanced Spontaneous Emission by Quantum Boxes in a Monolithic Optical Microcavity. Physical Review Letters, 81(5):1110-1113, August 1998.

[6] Z. Jacob, J. Y. Kim, G. V. Naik, A. Boltasseva, E. E. Narimanov, and V. M. Shalaev. Engineering photonic density of states using metamaterials. Applied Physics B, 100(1):215-218, June 2010.

[7] Z. Jacob, L. V. Alekseyev, and E. Narimanov. Semiclassical theory of the hyperlens. Journal of the Optical Society of America. A, Optics, image science, and vision, 24(10):A52-9, October 2007.

[8] J. D. Jackson. Classical Electrodynamics. Wiley, 3rd edition, 2007.

[9] Z. Jacob, L. V. Alekseyev, and E. Narimanov. Optical Hyperlens: Far-field imaging beyond the diffraction limit. Optics express, 14(18):8247-56, September 2006.

[10] R. Abermann, R. Kramer, and J. Maser. Structure and internal stress in ultra-thin silver films deposited on MgF2 and $\mathrm{SiO}$ substrates. Thin Solid Films, 52(2):215-229, July 1978.

[11] J. R. Lakowicz. Radiative decay engineering 3. Surface plasmoncoupled directional emission. Analytical Biochemistry, 324(2):153169, January 2004.

[12] J. R. Lakowicz. Radiative decay engineering: biophysical and biomedical applications. Analytical biochemistry, 298(1):1-24, November 2001. 